\title{
Communications
}

\section{Examen de spécialiste \\ Examen de spécialiste en vue de l'obtention de la formation approfondie en cytopathologie à adjoindre au titre de spécialiste en pathologie}

Lieu: Institut de pathologie de l'Université de Berne.

\section{Date: Printemps: le 10 mai 2016.}

\section{Délai d'inscription: le 31 janvier 2016}

Vous trouverez de plus amples informations sur le site web de l'ISFM www.siwf.ch $\rightarrow$ Domaines spécialisés $\rightarrow$ Titres de spécialiste et formations approfondies (formation postgraduée) $\rightarrow$ pathologie

Collège de médecine de premier recours CMPR

\section{Dates des Cours pour l'attestation de} formation complémentaire du laboratoire du praticien (AFC-LP) 2016

a) Kurse à 2 Tage auf Deutsch (Voraussetzung: absolviertes e-Learning, Test bestanden)

Kursort: Luzern

Daten für 2 Tage Präsenzkurs:

- 26./27. April 2016

- 28./29. April 2016

- 22./23. November 2016

- 24./25. November 2016

b) Cours à 2 jours en français

(condition: suivre le e-learning avec réussite):

Lieu: Lausanne

Date: 23/24 juin 2016

c) Cours à 3 jours en italien (sans e-learning)

Lieu: Locarno

Date: 24/25/26 novembre 2016

Important: Nous nous réservons le droit de biffer un cours s'il n'y a pas assez d'inscriptions.

Inscription: Consultez, s.v.p., les infos sur www.kollegium.ch/aus/plf.html. Remplissez là le formulaire d'inscription et envoyez-le au secrétariat du CMPR, à l'une des adresses suivantes: Secrétariat du CMPR, Rue de l'Hôpital 15, case postale 1552, 1701 Fribourg; courriel khm[at]hin.ch.

Vous recevrez la facture du cours et de l'attestation de formation, de même que l'invitation au cours de votre choix.

\section{Académie Suisses des}

Sciences Médicales

Recherche avec l'être humain Guide pratique

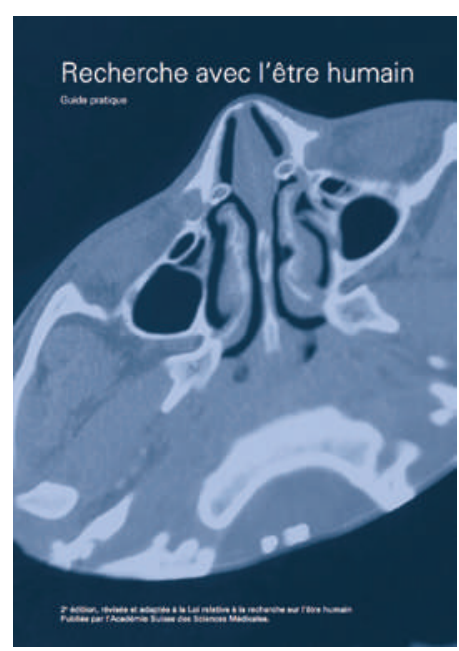

La version actualisée du guide pratique Recherche avec l'être humain est disponible. La révision a notamment tenu compte des nouvelles conditions juridiques en vigueur depuis l'introduction de la nouvelle loi relative à la recherche sur l'être humain, le

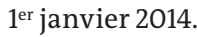

Le guide pratique offre un soutien concret lors de la planification, de la réalisation et de l'évaluation de projets de recherche avec des êtres humains dans le domaine de la santé et de la maladie. Il donne un aperçu schématique des notions de base de la recherche et des conditions cadres juridiques. Par ailleurs, les nombreuses questions éthiques soulevées par la recherche avec les êtres humains et l'utilisation de leurs données personnelles sont abordées dans ce document.

Il s'adresse au premier chef aux chercheurs et aux membres des commissions d'éthique de la recherche ainsi qu'aux médecins et aux professionnels de la santé qui ne mènent pas eux-mêmes des projets de recherche, mais sont en charge de patients participant à des études. Les politiciens, les collaborateurs des médias ou toutes les personnes intéressées par la médecine et la recherche sont également ciblés.

La Swiss Clinical Trial Organisation (SCTO), en tant que plateforme centrale de coopération de la recherche clinique orientée vers le patient en Suisse, et l'organisation faîtière des commissions d'éthique suisses (swissethics) soutiennent le guide pratique et le recommandent comme outil de travail. Il est disponible sur le site Internet de l'ASSM en français, allemand et anglais sous «Publications» dans le menu «Guides pratiques». Des exemplaires imprimés en français ou en allemand peuvent être commandés au secrétariat général de l'ASSM: mail[at]samw.ch.

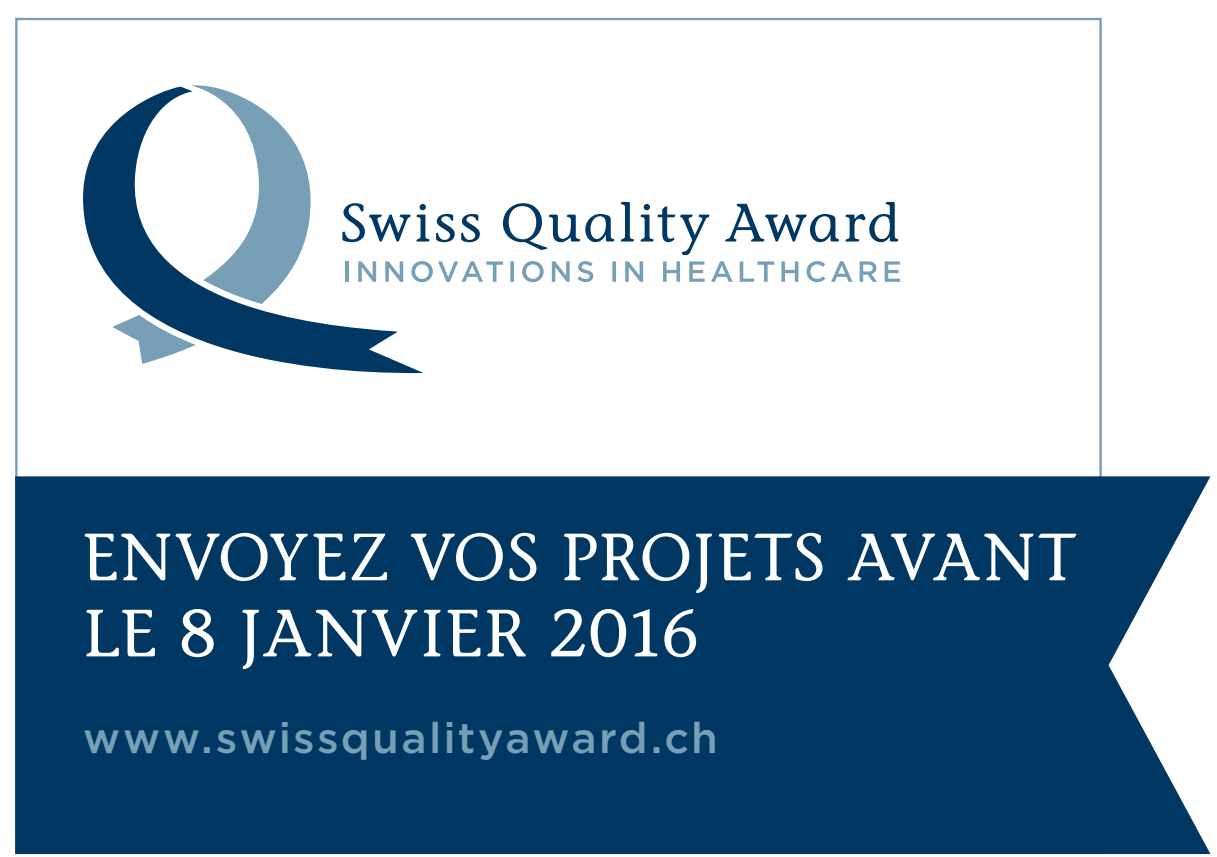

